\title{
Masses of vector bosons in two-color dense QCD based on the hidden local symmetry
}

\author{
Masayasu Harada, Chiho Nonaka, and Tetsuro Yamaoka \\ Department of Physics, Nagoya University, Nagoya, 464-8602, Japan
}

(Dated: June 7, 2018)

\begin{abstract}
We construct a low energy effective Lagrangian for the two-color QCD including the "vector" bosons (mesons with $J^{P}=1^{-}$and diquark baryons with $J^{P}=1^{+}$) in addition to the pseudo Nambu-Goldstone bosons with a degenerate mass $M_{\pi}$ (mesons with $J^{P}=0^{-}$and baryons with $J^{P}=0^{+}$) based on the chiral symmetry breaking pattern of $S U\left(2 N_{f}\right) \rightarrow S p\left(2 N_{f}\right)$ in the framework of the hidden local symmetry. We investigate the dependence of the "vector" boson masses on the baryon number density $\mu_{B}$. We show that the $\mu_{B}$-dependence signals the phase transition of $U(1)_{B}$ breaking. We find that it gives information about mixing among "vector" bosons: e.g. the mass difference between $\rho$ and $\omega$ mesons is proportional to the mixing strength between the diquark baryon with $J^{P}=1^{+}$and the anti-baryon. We discuss the comparison with lattice data for two-color QCD at finite density.

PACS numbers: 11.30.Rd, 12.39.Fe, 05.70.Fh
\end{abstract}

\section{INTRODUCTION}

Quantum chromodynamics (QCD) shows various phases under extreme conditions. At very high temperature and/or density, the phase structure can be studied with perturbative approaches. However, it is difficult to study it especially near the critical temperature and/or density directly from QCD, because of the strong coupling. Lattice QCD simulation is one of powerful theoretical tools, but it is not applicable in the finite density region due to the sign problem [1]. The problem in simulations of real-life QCD at finite baryon density produces interest in the two-color QCD with quarks in the fundamental representation [2, 3], the QCD with quarks in the adjoint representation [3, 4], and so on, that are free from the sign problem. In particular, two-color QCD has some interesting features in the following respect: Color-singlet baryons appear together with ordinary mesons as Nambu-Goldstone 
(NG) bosons associated with the spontaneous breaking of the chiral symmetry and their interactions are determined uniquely by the low-energy theorem. This allows us to construct low-energy effective theories including the baryons as light degrees of freedom naturally, and to investigate properties of hadrons even at finite baryon density using them. Furthermore, because there are many studies of the lattice QCD simulations, we can make a comparison of results from effective theories with those from the lattice analyses [5 7 ].

Actually, in two-color QCD, the phase structure at finite baryon number density $\mu_{B}$ was studied [3] using the chiral Lagrangian including the pseudo-NG bosons, having masses of $M_{\pi}$, which are associated with the chiral symmetry breaking of $S U\left(2 N_{f}\right) \rightarrow S p\left(2 N_{f}\right)$. It was shown that the phase transition from the symmetric phase of the baryon number $U(1)_{B}$ to the broken phase takes place at $\mu_{B}=M_{\pi}$, triggered by the condensation of the baryonic pseudo-NG bosons. There, the density dependences of the masses of pseudo-NG bosons are also studied and it was found that a baryonic boson becomes the massless NG boson in the $U(1)_{B}$ broken phase.

The density dependences of "vector" bosons (In this paper we call "vector" bosons which consist of mesons with $J^{P}=1^{-}$and diquark baryons with $J^{P}=1^{+}$.) are studied in the lattice simulation [6, 7]. It was shown that the mass of " $\rho$ " meson decreases with $\mu_{B}$ [6, 7], and that the mass of anti-baryon with $J^{P}=1^{+}$increases linearly with $\mu_{B}$ for $\mu_{B} \lesssim M_{\pi}$ while that of baryon decreases linearly [7]. For $\mu_{B} \gtrsim M_{\pi}$, the baryon mass is not yet clearly confirmed. On the other hand, the behaviors of the masses were also studied in an effective model [8]. It is interesting to study the masses in a general effective model which can include several models with the parameters chosen suitably.

In this paper we construct a low energy effective Lagrangian for the two-color QCD including the "vector" bosons (mesons with $J^{P}=1^{-}$and diquark baryons with $J^{P}=1^{+}$) in addition to the pseudo-NG bosons (mesons with $J^{P}=0^{-}$and baryons with $J^{P}=0^{+}$). The effective Lagrangian is composed based on the chiral symmetry breaking pattern of $S U\left(2 N_{f}\right) \rightarrow S p\left(2 N_{f}\right)$ in the framework of the hidden local symmetry (HLS) [9 11], and the "vector" bosons are introduced as the gauge bosons of the $\operatorname{Sp}\left(2 N_{f}\right)$ HLS. The HLS is equivalent to other models for "vector" bosons such as the CCWZ matter field [12], the tensor field [13] and the Massive Yang-Mills field [14]. Furthermore, it is possible to perform the systematic derivative expansion in the HLS [11, 15-17] , which allows us to study the parameter dependences of the masses at finite density in a systematic way. 
We study the vacuum structure of the model in the case of $N_{f}=2$ using the leading order Lagrangian, and show that the flavor-singlet " $\omega$ "-meson carrying $J^{P}=1^{-}$has a vacuum expectation value in the time component. As a result, the phase structure is the same as the one determined by including only the pseudo-NG bosons [3]: For $\mu_{B}>M_{\pi}$, a baryonic pseudo-NG boson $\left(J^{P}=0^{+}\right.$state $)$condenses, which causes the spontaneous breaking of the baryon number symmetry, $U(1)_{B}$. We show that the mass of the anti-baryon (baryon) with $J^{P}=1^{+}$increases (decreases) for $\mu_{B}<M_{\pi}$ and turns to decrease (increase) for $\mu_{B}>M_{\pi}$. These behaviors signal the phase transition of $U(1)_{B}$ breaking. The effect of higher order terms is shown to make $\rho$ and $\omega$ meson masses decrease for $\mu_{B}>M_{\pi}$ consistently with lattice data [6, 7]. Furthermore, the mass difference between $\rho$ and $\omega$ mesons is proportional to the mixing strength between the diquark baryon with $J^{P}=1^{+}$and the anti-baryon.

The paper is organized as follows. In section 2 we construct the chiral Lagrangian based on the HLS. The vacuum structure and the $\mu_{B}$-dependences of the masses are studied at the leading order in section 3 . In section 4 we show the effects of higher order terms to the masses and mixings. Section 5 is devoted to a summary and discussions. Several intricate calculations and useful formulas are summarized in Appendices $\mathrm{A}-\mathrm{C}$.

\section{HLS MODEL IN TWO-COLOR QCD}

Let us construct a low energy effective Lagrangian including NG bosons associated with the spontaneous chiral symmetry breaking $S U\left(2 N_{f}\right) \rightarrow S p\left(2 N_{f}\right)$, following Ref. [3].

In the following we divide the hermitian generators, $\left\{T^{A}\right\}$ of $S U\left(2 N_{f}\right)$ normalized as $\operatorname{tr}\left[T^{A} T^{B}\right]=\delta^{A B} / 2$, into two classes: The generators of $S p\left(2 N_{f}\right)$ denoted by $\left\{S^{\alpha}\right\}$ with $\alpha=1, \ldots, 2 N_{f}^{2}+N_{f}$; and the remaining generators of $S U\left(2 N_{f}\right)$ by $\left\{X^{a}\right\}$ with $a=1, \ldots, 2 N_{f}^{2}-$ $N_{f}-1$. These generators satisfy the relations

$$
\left(S^{\alpha}\right)^{T}=\bar{\Sigma} S^{\alpha} \bar{\Sigma}, \quad\left(X^{a}\right)^{T}=-\bar{\Sigma} X^{a} \bar{\Sigma},
$$

where $\bar{\Sigma}$ is a $2 N_{f} \times 2 N_{f}$ matrix satisfying following properties:

$$
\bar{\Sigma}^{2}=-\mathbf{1}, \quad \bar{\Sigma}^{T}=\bar{\Sigma}^{\dagger}=-\bar{\Sigma} .
$$

The chiral symmetry breaking gives $2 N_{f}^{2}-N_{f}-1$ NG bosons $\pi$ which are encoded in the $2 N_{f} \times 2 N_{f}$ matrix as

$$
\Sigma=\xi(\pi) \bar{\Sigma} \xi^{T}(\pi)
$$


where

$$
\xi(\pi)=e^{i \pi / f_{\pi}}, \quad \pi=\pi^{a} X^{a},
$$

with the decay constant $f_{\pi}$. ${ }^{\# 1}$ Transformation property of $\xi(\pi)$ under the chiral symmetry is given by

$$
\xi(\pi) \rightarrow g \xi(\pi) h^{\dagger}(\pi, g), \quad\left(h \in S p\left(2 N_{f}\right), g \in S U\left(2 N_{f}\right)\right)
$$

From this together with the relations in Eq. (2.1), we see that $\Sigma$ transforms linearly under the chiral symmetry as

$$
\Sigma \rightarrow g \Sigma g^{T}
$$

The effective Lagrangian including the NG bosons should be invariant under the global $S U\left(2 N_{f}\right)$ group and under the Lorentz transformation, which is given by [3]

$$
\mathcal{L}=\frac{f_{\pi}^{2}}{4} \operatorname{tr}\left[\left(\partial_{\nu} \Sigma\right)\left(\partial^{\nu} \Sigma^{\dagger}\right)\right]
$$

Next, we include the "vector" boson fields \#2 into the Lagrangian based on the hidden local symmetry (HLS) 9 11]. We decompose the field $\Sigma$ as

$$
\Sigma=\xi_{L}^{\dagger} \bar{\Sigma} \xi_{L}^{T}
$$

where $\xi_{L}$ is given by

$$
\xi_{L}=\xi(\sigma) \xi^{\dagger}(\pi), \quad\left(\xi(\sigma)=e^{i \sigma / f_{\sigma}}, \quad \sigma=\sigma^{\alpha} S^{\alpha}\right)
$$

with $\sigma$ being the NG bosons associated with the spontaneous breaking of the HLS and $f_{\sigma}$ the corresponding decay constant. The transformation property of $\xi_{L}$ is given by

$$
\xi_{L} \rightarrow h \xi_{L} g^{\dagger}
$$

where

$$
h \in\left[S p\left(2 N_{f}\right)\right]_{\text {local }}, \quad g \in\left[S U\left(2 N_{f}\right)\right]_{\text {global }} .
$$

For constructing the HLS Lagrangian, it is convenient to introduce the field $\xi_{R}$ by

$$
\xi_{R}=\bar{\Sigma}\left(\xi_{L}^{\dagger}(x)\right)^{T} \bar{\Sigma}^{\dagger}=\xi(\sigma) \xi(\pi)
$$

\#1 Note that the NG bosons consist of the mesons with $J^{P}=0^{-}$and the (anti-) baryons with $J^{P}=0^{+}$.

\#2 Here the "vector" boson fields imply the meson fields with $J^{P}=1^{-}$and the (anti-) baryon fields with $J^{P}=1^{+}$. 
which transforms as

$$
\xi_{R} \rightarrow h \xi_{R}\left(\bar{\Sigma} g^{T} \bar{\Sigma}^{\dagger}\right)
$$

Note that, in the HLS, the entire symmetry $G_{\text {global }} \times H_{\text {local }}$ is spontaneously broken to its subgroup $H_{\text {global }}=\left[S p\left(2 N_{f}\right)\right]_{\text {global }}$, so that the NG bosons $\sigma=\sigma^{\alpha} S^{\alpha}$ are absorbed into the HLS gauge bosons. The basic quantities to construct the HLS Lagrangian are the following two Maurer-Cartan 1-forms:

$$
\begin{gathered}
\hat{\alpha}_{\perp \nu}=\left[\left(D_{\nu} \xi_{R}\right) \xi_{R}^{\dagger}-\left(D_{\nu} \xi_{L}\right) \xi_{L}^{\dagger}\right] /(2 i), \\
\hat{\alpha}_{\| \nu}=\left[\left(D_{\nu} \xi_{R}\right) \xi_{R}^{\dagger}+\left(D_{\nu} \xi_{L}\right) \xi_{L}^{\dagger}\right] /(2 i),
\end{gathered}
$$

where the covariant derivatives are read from the transformation properties in Eqs. (2.10) and (2.13) as

$$
\begin{aligned}
& D_{\nu} \xi_{L}=\partial_{\nu} \xi_{L}-i V_{\nu} \xi_{L}+i \xi_{L} G_{\nu} \\
& D_{\nu} \xi_{R}=\partial_{\nu} \xi_{R}-i V_{\nu} \xi_{R}+i \xi_{R}\left(\bar{\Sigma} G_{\nu}^{T} \bar{\Sigma}\right)
\end{aligned}
$$

with $V_{\nu}=V_{\nu}^{\alpha} S^{\alpha}$ and $G_{\nu}=G_{\nu}^{A} T^{A}$ being the $H_{\text {local gauge bosons and the external gauge }}$ bosons corresponding to the chiral symmetry (see Appendix A), respectively. These gauge bosons transform as

$$
\begin{gathered}
V_{\nu} \rightarrow V_{\nu}^{\prime}=h V_{\nu} h^{\dagger}+i h\left(\partial_{\nu} h^{\dagger}\right), \\
G_{\nu} \rightarrow G_{\nu}^{\prime}=g G_{\nu} g^{\dagger}+i g\left(\partial_{\nu} g^{\dagger}\right) .
\end{gathered}
$$

It should be noticed that in the HLS formalism we can introduce the "vector" bosons $V_{\nu}$ and the external chiral gauge bosons $G_{\nu}$ independently. Since the covariantized 1-forms $\hat{\alpha}_{\perp \nu}$ and $\hat{\alpha}_{\| \nu}$ in Eqs. (2.14) and (2.15) transform homogeneously:

$$
\hat{\alpha}_{\perp, \|}^{\nu} \rightarrow h \hat{\alpha}_{\perp, \|}^{\nu}(x) h^{\dagger}
$$

we have the following two invariants:

$$
f_{\pi}^{2} \operatorname{tr}\left[\hat{\alpha}_{\perp \nu} \hat{\alpha}_{\perp}^{\nu}\right], \quad f_{\sigma}^{2} \operatorname{tr}\left[\hat{\alpha}_{\| \nu} \hat{\alpha}_{\|}^{\nu}\right]
$$

We introduce the kinetic term of the "vector" bosons:

$$
-\frac{1}{2 g^{2}} \operatorname{tr}\left[V_{\nu \rho} V^{\nu \rho}\right]
$$


where $g$ is the HLS gauge coupling constant and $V_{\nu \rho}$ is the field strength defined by

$$
V_{\nu \rho} \equiv \partial_{\nu} V_{\rho}-\partial_{\rho} V_{\nu}-i\left[V_{\nu}, V_{\rho}\right]
$$

In addition, we include the external scalar and pseudoscalar source fields $\chi$ given in Eq. (A.14) into the Lagrangian. Note that the vacuum expectation value (VEV) of $\chi$ gives the explicit chiral symmetry breaking due to the current quark masses as

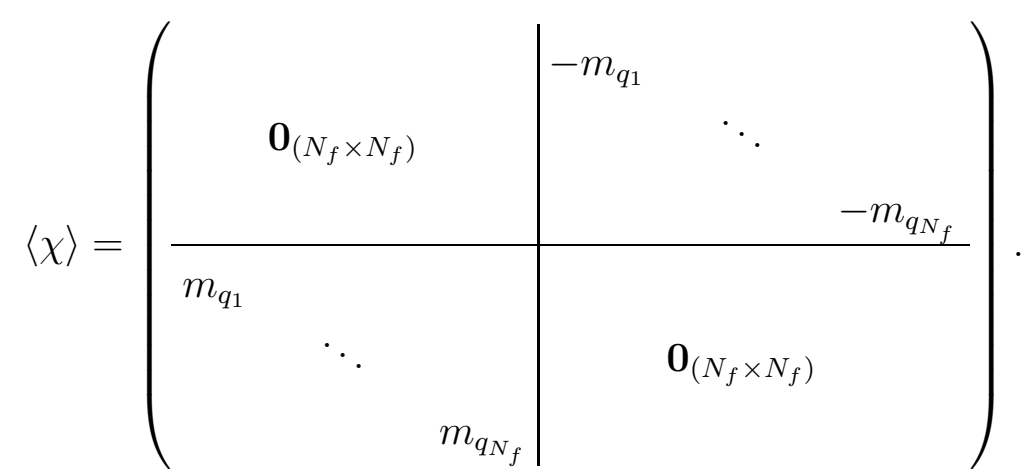

The $\chi$ transforms under the chiral symmetry as $\chi \rightarrow g^{*} \chi g^{\dagger}$. (see Eq. (A.16)) Since $\hat{\alpha}_{\| \nu}$ as well as $\hat{\alpha}_{\perp \nu}$ transforms homogeneously under the HLS, it is convenient to convert $\chi$ into a field $\hat{\chi}$ as

$$
\hat{\chi}=2 G \xi_{R} \bar{\Sigma} \chi \xi_{L}^{\dagger}
$$

where $G$ is a parameter carrying the mass dimension one. $\hat{\chi}$ in Eq. (2.25) transforms homogeneously under the HLS (see Eqs. (2.10) and (2.13) for the transformation properties of $\left.\xi_{L, R}\right)$ :

$$
\hat{\chi} \rightarrow h \hat{\chi} h^{\dagger}
$$

For convenience, we summarize the transformation properties of the building blocks under parity $(P)$, charge-conjugation $(C)$, and HLS in TABLE I. In this table, $\Sigma_{c}$ is the $2 N_{f} \times 2 N_{f}$ matrix defined as

$$
\Sigma_{c} \equiv\left(\begin{array}{cc}
\mathbf{0} & \mathbf{1}_{N} \\
-\mathbf{1}_{N} & \mathbf{0}
\end{array}\right),
$$

which determines the direction of the chiral condensate, i.e. how to embed the $\operatorname{Sp}\left(2 N_{f}\right)$ into $S U\left(2 N_{f}\right) . \Omega$ is the $2 N_{f} \times 2 N_{f}$ matrix given as

$$
\Omega \equiv\left(\begin{array}{cc}
\mathbf{0} & \mathbf{1}_{N} \\
\mathbf{1}_{N} & \mathbf{0}
\end{array}\right)
$$




\begin{tabular}{cccc}
\hline Building block & $P$ & $C$ & HLS \\
\hline$\hat{\alpha}_{\|}^{\nu}$ & $\left(\Omega \Sigma_{c}\right) \hat{\alpha}_{\| \nu}\left(\Omega \Sigma_{c}\right)$ & $-\hat{\alpha}_{\|}^{\nu T}$ & $h \hat{\alpha}_{\|}^{\nu} h^{\dagger}$ \\
$\hat{\alpha}_{\perp}^{\nu}$ & $-\left(\Omega \Sigma_{c}\right) \hat{\alpha}_{\perp \nu}\left(\Omega \Sigma_{c}\right)$ & $\hat{\alpha}_{\perp}^{\nu T}$ & $h \hat{\alpha}_{\perp}^{\nu} h^{\dagger}$ \\
$\hat{\chi}$ & $\left(\Omega \Sigma_{c}\right) \hat{\chi}^{\dagger}\left(\Omega \Sigma_{c}\right)$ & $\hat{\chi}^{T}$ & $h \hat{\chi}^{\dagger}$ \\
$G^{\nu}$ & $-\Omega G_{\nu}^{T} \Omega$ & $-\Sigma_{c} G^{\nu} \Sigma_{c}$ & $g G_{\nu} g^{\dagger}+i g\left(\partial_{\nu} g^{\dagger}\right)$ \\
\hline
\end{tabular}

TABLE I: Transformation properties of the building blocks under parity $(P)$, charge-conjugation $(C)$, and HLS.

Then the lowest order term invariant under the $P$ transformation is given by

$$
\frac{f_{\chi}^{2}}{4} \operatorname{tr}\left[\hat{\chi}+\hat{\chi}^{\dagger}\right]
$$

where $f_{\chi}$ is introduced to renormalize the quadratically divergent correction to this term [18]. In the present analysis, we introduced this parameter in such a way that the field $\hat{\chi}$ does not get any renormalization effect.

Finally, the HLS Lagrangian with leading order terms is given by

$$
\mathcal{L}=-\frac{1}{2 g^{2}} \operatorname{tr}\left[V_{\nu \rho} V^{\nu \rho}\right]+f_{\sigma}^{2} \operatorname{tr}\left[\hat{\alpha}_{\| \nu}^{2}\right]+f_{\pi}^{2} \operatorname{tr}\left[\hat{\alpha}_{\perp \nu}^{2}\right]+\frac{f_{\chi}^{2}}{4} \operatorname{tr}\left[\hat{\chi}+\hat{\chi}^{\dagger}\right] .
$$

\section{VACUUM STRUCTURE AND SPECTRUM OF "VECTOR" BOSONS AT NONZERO BARYON CHEMICAL POTENTIAL}

In this section, we study the vacuum structure and examine the effects of nonzero chemical potential for the baryon number charge on the spectrum of "vector" bosons in the case of $N_{f}=2$. In Table I, we show the fields included in the present model together with their quantum numbers for the $S U(2)$ "isospin" $I$ \#3 , the baryon number charge $B{ }^{\# 4}$ and the spin-parity $J^{P}$. The effect of chemical potential for the baryon number charge is introduced as the vacuum expectation value (VEV) of the external chiral gauge boson as

$$
\left\langle G_{\nu}\right\rangle=\delta_{0 \nu} \frac{\mu_{B}}{2}\left(\begin{array}{cc}
\mathbf{1} & \mathbf{0} \\
\mathbf{0} & -\mathbf{1}
\end{array}\right)=\frac{\mu_{B} \delta_{0 \nu}}{2} B
$$

\#3 For $N_{f}=2$ there exists an $S U(2)$ flavor symmetry which we call the "isospin" symmetry.

\#4 We follow the convention of baryon number given in Ref. 3], which is different from the one in Ref. 7]. 


\begin{tabular}{ccccc}
\hline Field & Generator & $I$ & $B$ & $J^{P}$ \\
\hline$\pi^{1,2,3}$ & $X^{1,2,3}$ & 1 & 0 & $0^{-}$ \\
$\pi_{B_{+}}=\left(\pi^{5}-i \pi^{4}\right) / \sqrt{2}$ & $\left(X^{5}-i X^{4}\right) / \sqrt{2}$ & 0 & +1 & $0^{+}$ \\
$\pi_{B_{-}}=\left(\pi^{5}+i \pi^{4}\right) / \sqrt{2}$ & $\left(X^{5}+i X^{4}\right) / \sqrt{2}$ & 0 & -1 & $0^{+}$ \\
$\rho^{1,2,3}=V^{1,2,3}$ & $S^{1,2,3}$ & 1 & 0 & $1^{-}$ \\
$\omega=V^{4}$ & $S^{4}$ & 0 & 0 & $1^{-}$ \\
$V_{B_{+}}=\left(V^{\alpha}+i V^{\beta}\right) / \sqrt{2}$ & $\left(S^{\alpha}+i S^{\beta}\right) / \sqrt{2}$ & 1 & +1 & $1^{+}$ \\
$V_{B_{-}}=\left(V^{\alpha}-i V^{\beta}\right) / \sqrt{2}$ & $\left(S^{\alpha}-i S^{\beta}\right) / \sqrt{2}$ & 1 & -1 & $1^{+}$ \\
\hline
\end{tabular}

TABLE II: Fields corresponding to the mass eigenstates at $\mu_{B}=0$, together with the "isospin" $I$, the baryon number charge $B$ and the spin-parity $J^{P}$. Indexes of the "vector" bosons with $J^{P}=1^{+}$ are taken as $(\alpha, \beta)=\{(5,6),(7,8),(9,10)\}$.

where $\mathbf{1}$ is the $2 \times 2$ unit matrix and $\mathbf{0}=\left(\begin{array}{ll}0 & 0 \\ 0 & 0\end{array}\right)$. In the following we restrict ourselves to the case where two quarks have the same current quark masses: The VEV of the external scalar field in Eq. (2.25) takes

$$
\langle\chi\rangle=m_{q}\left(\begin{array}{cc}
\mathbf{0} & -\mathbf{1} \\
\mathbf{1} & \mathbf{0}
\end{array}\right)=m_{q} \hat{M}
$$

Here we assume that the spatial rotational symmetry is not broken, so that the relevant VEVs of the fields in the unitary gauge of the HLS, $\sigma=0$, are reduced to

$$
\left\langle\xi_{L}\right\rangle=\xi^{\dagger}(\tilde{\pi}), \quad\left\langle\xi_{R}\right\rangle=\xi(\tilde{\pi}), \quad\left\langle V_{\nu}(x)\right\rangle=\tilde{V}_{\nu}=\left(\tilde{V}_{0}, \mathbf{0}\right)
$$

Replacing the fields with three VEVs as above, we obtain the static potential as

$$
\begin{aligned}
V_{\text {potential }} & =-f_{\sigma}^{2} \operatorname{tr}\left[\left\{\tilde{V}_{0}-\frac{\mu_{B}}{2}\left(\xi^{\dagger}(\tilde{\pi}) B \xi(\tilde{\pi})+\xi(\tilde{\pi}) B \xi^{\dagger}(\tilde{\pi})\right)\right\}^{2}\right] \\
& -\frac{f_{\pi}^{2} \mu_{B}^{2}}{16} \operatorname{tr}\left[\left\{\xi^{\dagger}(\tilde{\pi}) B \xi(\tilde{\pi})-\xi(\tilde{\pi}) B \xi^{\dagger}(\tilde{\pi})\right\}^{2}\right]-\frac{M_{\pi}^{2} f_{\pi}^{2}}{4} \operatorname{tr}\left[\xi^{2}(\tilde{\pi}) \bar{\Sigma} \hat{M}+(\text { h.c })\right],
\end{aligned}
$$

where we use $B^{T}=B$ and $M_{\pi}$ is the mass of $\pi$ s defined as

$$
M_{\pi}^{2}=\frac{2 G m_{q} f_{\chi}^{2}}{f_{\pi}^{2}}
$$

From the stationary condition for $\tilde{V}_{0}$, we obtain

$$
\tilde{V}_{0}=\frac{\mu_{B}}{2}\left(\xi^{\dagger}(\tilde{\pi}) B \xi(\tilde{\pi})+\xi(\tilde{\pi}) B \xi^{\dagger}(\tilde{\pi})\right) .
$$




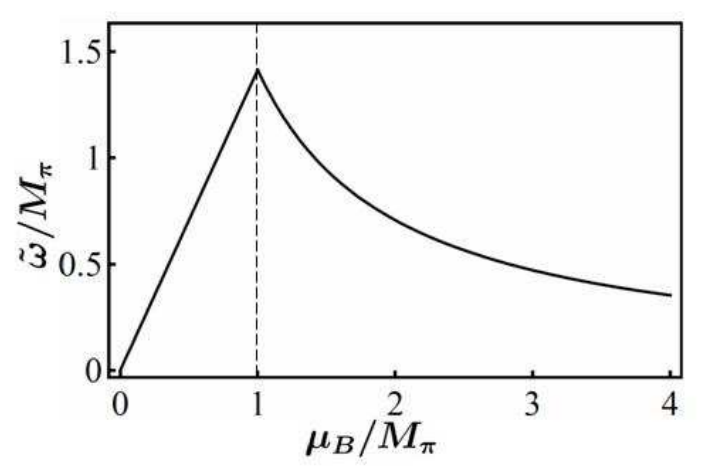

FIG. 1: $\mu_{B}$-dependence of the VEV $\tilde{\omega}$ in unit of $M_{\pi}$ as a function of $\mu_{B} / M_{\pi}$.

Substituting this into $V_{\text {potential, }}$, we have

$$
\left.V_{\text {potential }}\right|_{\tilde{V}_{0}}=-\frac{f_{\pi}^{2}}{4} \operatorname{tr}\left[\frac{\mu_{B}^{2}}{4}\left\{\xi^{\dagger}(\tilde{\pi}) B \xi(\tilde{\pi})-\xi(\tilde{\pi}) B \xi^{\dagger}(\tilde{\pi})\right\}^{2}+M_{\pi}^{2}\left\{\xi^{2}(\tilde{\pi}) \bar{\Sigma} \hat{M}+(\text { h.c })\right\}\right] .
$$

This is equivalent to the potential term of the chiral Lagrangian for NG bosons analyzed in Ref. [3]. Then, the value of $\xi(\tilde{\pi})$ which minimizes the potential is obtained as

$$
\xi(\tilde{\pi})=e^{i \tilde{\pi}^{5} X^{5}}
$$

where $X^{5}$ is given in Eqs. (B.6) and (B.7) and the VEV $\tilde{\pi}^{5}$ is determined as

$$
\tilde{\pi}^{5}=\sqrt{2} f_{\pi} \theta
$$

with

$$
\theta=0, \quad\left(\text { for } 0<\mu_{B}<M_{\pi}\right), \quad \cos \theta=\frac{M_{\pi}^{2}}{\mu_{B}^{2}}, \quad\left(\text { for } \mu_{B}>M_{\pi}\right) .
$$

This means that there is a condensation of the baryonic-NG boson with $J^{P}=0^{+}$and $U(1)_{B}$ symmetry is broken spontaneously for $\mu_{B}>M_{\pi}$. By substituting this VEV into Eq. (3.6), the VEV of the "vector" boson fields $\tilde{V}_{0}^{\alpha}$ is determined as

$$
\tilde{V}_{0}^{\alpha}= \begin{cases}\sqrt{2} \mu_{B} \cos \theta, & (\alpha=4) \\ 0, & (\alpha \neq 4)\end{cases}
$$

This implies that the time component of $\omega$ meson (see Table II) has a VEV for any $\mu_{B}$, as in the ordinary three-color QCD. In FIG. 1, we plot the $\mu_{B}$-dependence of $\tilde{\omega}=\tilde{V}_{0}^{4}$. This shows that the $\tilde{\omega}$ increases with $\mu_{B}$ for $\mu_{B}<M_{\pi}$ while it decreases for $\mu_{B}>M_{\pi}$. 
We define the masses of "vector" bosons as the energies determined from the zero momentum limit of the dispersion relation. We shift the "vector" boson fields as

$$
V_{\nu}(x) \rightarrow V_{\nu}(x)+\tilde{V}_{\nu}
$$

and retain quadratic terms only in Eq. (2.30). Performing the Fourier transformation and taking the low-momentum limit yields

$$
\begin{aligned}
& \int d^{4} x\left[-\frac{1}{2 g^{2}} \operatorname{tr}\left(V_{\nu \rho} V^{\nu \rho}\right)+f_{\sigma}^{2} \operatorname{tr}\left(V_{\nu} V^{\nu}\right)\right] \\
& \rightarrow \frac{1}{2 g^{2}} \hat{V}_{j}^{\alpha}(-E)\left[E^{2} \delta^{\alpha \beta}-2 i f^{\alpha \beta \gamma}\left(\tilde{V}_{0}\right)^{\gamma} E+f^{\alpha \gamma \theta} f^{\beta \delta \gamma}\left(\tilde{V}_{0}\right)^{\gamma}\left(\tilde{V}_{0}\right)^{\delta}-M_{V}^{2} \delta^{\alpha \beta}\right] \hat{V}_{j}^{\beta}(E) \\
& \equiv \hat{V}_{j}^{\alpha} \Gamma^{\alpha \beta} \hat{V}_{j}^{\beta},
\end{aligned}
$$

where $f^{\alpha \beta \gamma}$ is a structure constant of $S p(4), M_{V}$ is the mass of "vector" boson at $\mu_{B}=0$;

$$
M_{V} \equiv g f_{\sigma}
$$

and $\hat{V}$ is defined as

$$
V_{\nu}^{\alpha}(x)=\int \frac{d^{4} k}{(2 \pi)^{4}} \hat{V}_{\nu}^{\alpha}(k) e^{-i k x}
$$

Using the basis of $S p(4)$ generators shown in Appendix $\mathrm{B}$, one finds that the matrix $\Gamma$ for the inverse propagator at zero momentum limit is block diagonal with four $1 \times 1$ terms and three $2 \times 2$ blocks. The four diagonal $1 \times 1$ terms are composed of $\hat{V}^{1}, \hat{V}^{2}, \hat{V}^{3}$ corresponding to the $\rho$ meson and $\hat{V}^{4}$ corresponding to the $\omega$ meson. The masses of these states are obtained as

$$
m_{\rho, \omega}=M_{V}
$$

Three $2 \times 2$ blocks for $\left(\hat{V}^{5}, \hat{V}^{6}\right),\left(\hat{V}^{7}, \hat{V}^{8}\right)$ and $\left(\hat{V}^{9}, \hat{V}^{10}\right)$ are identical because of the "isospin" symmetry. The $2 \times 2$ block for $\hat{V}^{5}$ and $\hat{V}^{6}$ is obtained as

$$
\left(\hat{V}_{j}^{5 \dagger} \hat{V}_{j}^{6 \dagger}\right)\left(\begin{array}{cc}
E^{2}-M_{V}^{2}+\mu_{B}^{2} \cos ^{2} \theta & 2 i \mu_{B} E \cos \theta \\
-2 i \mu_{B} E \cos \theta & E^{2}-M_{V}^{2}+\mu_{B}^{2} \cos ^{2} \theta
\end{array}\right)\left(\begin{array}{c}
\hat{V}_{j}^{5} \\
\hat{V}_{j}^{6}
\end{array}\right) .
$$

This matrix is diagonalized by the fields $V_{B_{+}}$and $V_{B_{-}}$defined in Table $\llbracket$ as

$$
\left(\hat{V}_{B_{+}, j}^{\dagger} \hat{V}_{B_{-}, j}^{\dagger}\right)\left(\begin{array}{cc}
\left(E+\mu_{B} \cos \theta\right)^{2}-M_{V}^{2} & 0 \\
0 & \left(E-\mu_{B} \cos \theta\right)^{2}-M_{V}^{2}
\end{array}\right)\left(\begin{array}{l}
\hat{V}_{B_{+}, j} \\
\hat{V}_{B_{-}, j}
\end{array}\right) .
$$




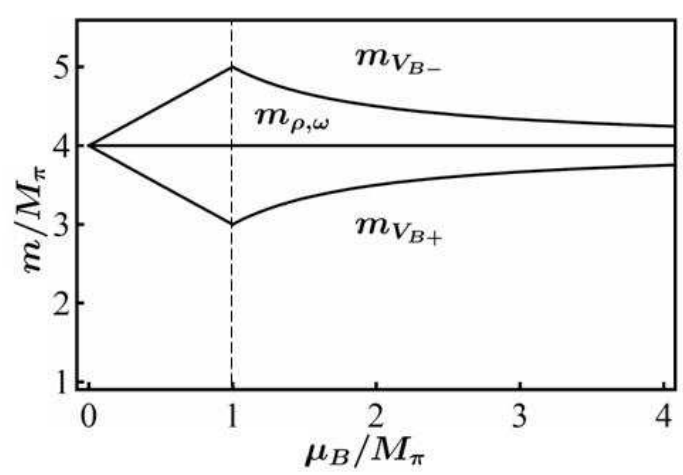

FIG. 2: The masses of the "vector" bosons in unit of $M_{\pi}$ as a function of $\mu_{B} / M_{\pi}$. We use $M_{V} / M_{\pi}=4$ and $g=1$ to make the plot.

From this, we obtain the masses as

$$
m_{V_{B+}}=M_{V}-\mu_{B} \cos \theta, \quad m_{V_{B-}}=M_{V}+\mu_{B} \cos \theta
$$

where we assumed $M_{V}>M_{\pi}$, so that $M_{V}>\mu_{B} \cos \theta$.

Let us consider the $\mu_{B}$-dependences of the masses of the "vector" bosons. Equation (3.16) indicates that the ordinary vector mesons with $J^{P}=1^{-}$do not change their masses at all. On the other hand, from Eq. (3.19) together with Eq. (3.10), we find that the mass of the baryon $\left(V_{B_{+}}\right)$with $J^{P}=1^{+}$decreases for $\mu_{B}<M_{\pi}$ and turns to increase for $\mu_{B}>M_{\pi}$, and the mass of anti-baryon $\left(V_{B_{-}}\right)$with $J^{P}=1^{+}$shows the opposite behavior. This indicates that the phase transition can be observed by seeing the masses of baryons with $J^{P}=1^{+}$. We stress that, for any value of $\mu_{B}$, the mass eigenstates are given by $V_{B_{+}}$and $V_{B_{-}}$, which are nothing but the eigenstates of the baryon number; $V_{B_{+}}$carries $B=+1$ and $V_{B_{-}}$does $B=-1$. In other words, the baryon with $J^{P}=1^{+}$does not mix with the anti-baryon having the same spin and parity, even though the baryon number $U(1)_{B}$ symmetry is spontaneously broken for $\mu_{B}>M_{\pi}$. Note that this feature holds only at the leading order: The mixing will generally appear when we include the higher order terms (see the next section). In FIG. 2, we plot the masses of "vector" bosons described in Eqs. (3.16) and (3.19) for $M_{V} / M_{\pi}=4$ as an example. In this figure, we can see the $\mu_{B}$-dependence of the masses of the "vector" bosons explained above. 


\section{EFFECT OF HIGHER ORDER TERMS}

In this section, we consider the effects of higher order terms in the hidden local symmetry (HLS). In QCD with three colors it is known that, thanks to the gauge invariance of the HLS, we can perform the systematic derivative expansion with including vector mesons in addition to the pseudo Nambu-Goldstone bosons when the masses of vector mesons are lighter than the chiral symmetry breaking scale (the chiral perturbation theory with the HLS [11, $15-17])$.

We adopt the same counting rule in the present case. Generally, there are 32 terms in the $\mathcal{O}\left(p^{4}\right)$ HLS Lagrangian [11, 17]. We present a complete list of $\mathcal{O}\left(p^{4}\right)$ Lagrangian in Appendix C. Here we include only the terms which do not alter the vacuum structure given in Eq. (3.7), neglecting the effect of current quark masses at $\mathcal{O}\left(p^{4}\right)$. In this case we have only three combinations which give corrections to the "vector" boson masses:

$$
\begin{aligned}
\mathcal{L}_{(4)_{1}}= & \bar{y}_{1} \operatorname{tr}\left[\hat{\alpha}_{\perp \mu} \hat{\alpha}_{\| \nu} \hat{\alpha}_{\perp}^{\mu} \hat{\alpha}_{\|}^{\nu}-\hat{\alpha}_{\perp \mu} \hat{\alpha}_{\perp}^{\mu} \hat{\alpha}_{\| \nu} \hat{\alpha}_{\|}^{\nu}+\hat{\alpha}_{\perp \mu} \hat{\alpha}_{\perp \nu} \hat{\alpha}_{\|}^{\mu} \hat{\alpha}_{\|}^{\nu}\right. \\
& \left.-\frac{1}{2}\left(\hat{\alpha}_{\perp \mu} \hat{\alpha}_{\|}^{\mu} \hat{\alpha}_{\perp \nu} \hat{\alpha}_{\|}^{\nu}+\hat{\alpha}_{\perp \mu} \hat{\alpha}_{\| \nu} \hat{\alpha}_{\perp}^{\nu} \hat{\alpha}_{\|}^{\mu}\right)\right], \\
\mathcal{L}_{(4)_{2}}= & \bar{y}_{2} \operatorname{tr}\left[\hat{\alpha}_{\perp \mu} \hat{\alpha}_{\| \nu} \hat{\alpha}_{\perp}^{\mu} \hat{\alpha}_{\|}^{\nu}-\hat{\alpha}_{\perp \mu} \hat{\alpha}_{\perp \nu} \hat{\alpha}_{\|}^{\nu} \hat{\alpha}_{\|}^{\mu}+\hat{\alpha}_{\perp \mu} \hat{\alpha}_{\perp \nu} \hat{\alpha}_{\|}^{\mu} \hat{\alpha}_{\|}^{\nu}\right. \\
& \left.-\frac{1}{2}\left(\hat{\alpha}_{\perp \mu} \hat{\alpha}_{\|}^{\mu} \hat{\alpha}_{\perp \nu} \hat{\alpha}_{\|}^{\nu}+\hat{\alpha}_{\perp \mu} \hat{\alpha}_{\| \nu} \hat{\alpha}_{\perp}^{\nu} \hat{\alpha}_{\|}^{\mu}\right)\right], \\
\mathcal{L}_{(4)_{3}}= & \bar{y}_{3} \operatorname{tr}\left[\hat{\alpha}_{\perp \mu} \hat{\alpha}_{\| \nu} \hat{\alpha}_{\perp}^{\mu} \hat{\alpha}_{\|}^{\nu}-\hat{\alpha}_{\perp \mu} \hat{\alpha}_{\perp}^{\mu} \hat{\alpha}_{\| \nu} \hat{\alpha}_{\|}^{\nu}+\hat{\alpha}_{\perp \mu} \hat{\alpha}_{\perp \nu} \hat{\alpha}_{\|}^{\nu} \hat{\alpha}_{\|}^{\mu}\right. \\
& \left.-\frac{1}{2}\left(\hat{\alpha}_{\perp \mu} \hat{\alpha}_{\|}^{\mu} \hat{\alpha}_{\perp \nu} \hat{\alpha}_{\|}^{\nu}+\hat{\alpha}_{\perp \mu} \hat{\alpha}_{\| \nu} \hat{\alpha}_{\perp}^{\nu} \hat{\alpha}_{\|}^{\mu}\right)\right],
\end{aligned}
$$

where $\bar{y}_{1}, \bar{y}_{2}$ and $\bar{y}_{3}$ are coefficients not determined by the HLS.

We consider the case of $N_{f}=2$ in the following analysis. From the vacuum expectation values (VEVs) of $\xi(\pi)$ and $V_{\nu}$ given in Eqs. (3.8) and (3.11), VEVs of $\hat{\alpha}_{\| \mu}$ and $\hat{\alpha}_{\perp \mu}$ are determined as

$$
\left\langle\hat{\alpha}_{\| \mu}\right\rangle=0, \quad\left\langle\hat{\alpha}_{\perp \mu}\right\rangle=\sqrt{2} \mu_{B} \delta_{\mu 0} \sin \theta X^{4}
$$

where $X^{4}$ is the fourth component of the broken generators given in Eq. (B.6). Substituting the $\operatorname{VEV}\left\langle\hat{\alpha}_{\perp \mu}\right\rangle$ into Eqs. (4.1)-(4.3) we obtain the correction to the masses of "vector" bosons as

$$
\mathcal{L}_{(4)_{1}}+\mathcal{L}_{(4)_{2}}+\mathcal{L}_{(4)_{3}} \stackrel{\mathcal{O}\left(V_{j}^{2}\right)}{\rightarrow} 2 \mu_{B}^{2} \sin ^{2} \theta\left[C_{1} \operatorname{tr}\left(X^{4} V_{j} X^{4} V^{j}\right)-C_{2} \operatorname{tr}\left(X^{4} X^{4} V_{j} V^{j}\right)\right]
$$



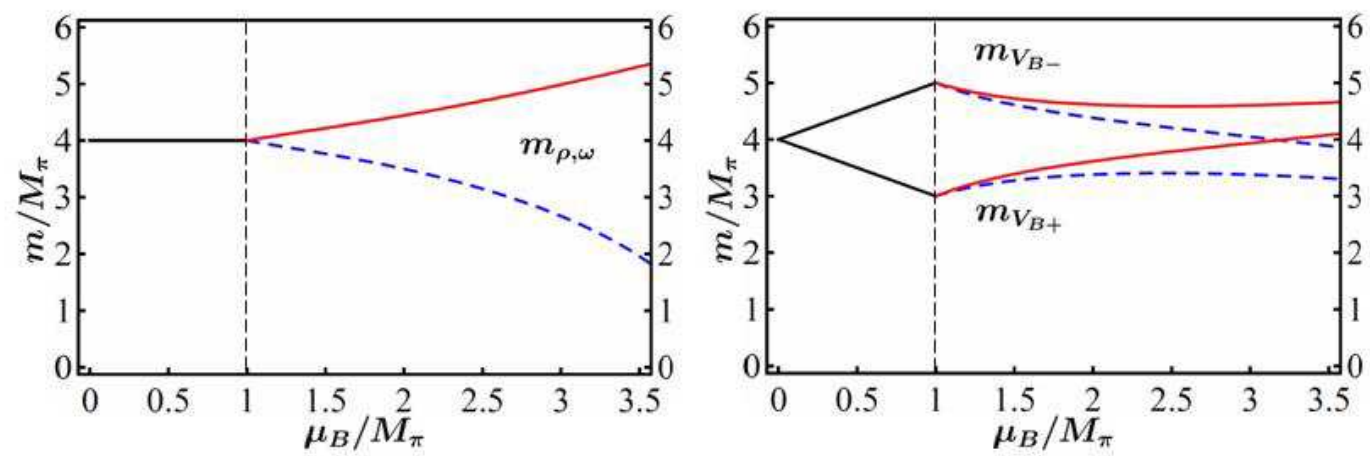

FIG. 3: $\mu_{B}$-dependences of the "vector" boson masses. The curves on the left figure are for the degenerate $\rho$ and $\omega$ mesons and those on the right figure are for baryonic and anti-baryonic "vector" bosons. We use $M_{V} / M_{\pi}=4, C_{1}=0, C_{2}= \pm 4$ and $g=1$ to make the curves. Dashed (blue) curves stand for $C_{2}=4$. Solid (red) curves stand for $C_{2}=-4$.

where $C_{1}$ and $C_{2}$ are certain linear combinations of $\bar{y}_{1}, \bar{y}_{2}$ and $\bar{y}_{3}$. Applying the same procedure as that in section 3 with the correction in Eq. (4.5), we obtain the masses of $\rho$ meson and $\omega$ meson as

$$
\begin{aligned}
& m_{\rho}=\sqrt{M_{V}^{2}+\frac{C_{1}-C_{2}}{4}\left(g \mu_{B} \sin \theta\right)^{2}}, \\
& m_{\omega}=\sqrt{M_{V}^{2}-\frac{C_{1}+C_{2}}{4}\left(g \mu_{B} \sin \theta\right)^{2}} .
\end{aligned}
$$

The quadratic term for $\left(V_{B_{+}}, V_{B_{-}}\right)$is obtained as

$$
\left(\hat{V}_{B_{+}}^{\dagger} \hat{V}_{B_{-}}^{\dagger}\right)\left(\begin{array}{cc}
\left(E+\mu_{B} \cos \theta\right)^{2}-M_{V}^{2}+\frac{C_{2}}{16}\left(g \mu_{B} \sin \theta\right)^{2} & \frac{C_{1}}{16}\left(g \mu_{B} \sin \theta\right)^{2} \\
\frac{C_{1}}{16}\left(g \mu_{B} \sin \theta\right)^{2} & \left(E-\mu_{B} \cos \theta\right)^{2}-M_{V}^{2}+\frac{C_{2}}{16}\left(g \mu_{B} \sin \theta\right)^{2}
\end{array}\right)\left(\begin{array}{c}
\hat{V}_{B_{+}} \\
\hat{V}_{B_{-}}
\end{array}\right) .
$$

The masses of "vector" bosons with $J^{P}=1^{+}$are obtained by diagonalizing the mass matrix in Eq. (4.8). We can see that the mixing between $V_{B_{+}}$and $V_{B_{-}}$is related with the mass difference between $m_{\rho}$ and $m_{\omega}$ as

$$
m_{\rho}^{2}-m_{\omega}^{2}=\frac{C_{1}}{2}\left(g \mu_{B} \sin \theta\right)^{2} .
$$

Equations (4.6), (4.7) and (4.8) show that the corrections to the masses from $\mathcal{O}\left(p^{4}\right)$ terms include the factor of $\sin \theta$, which is zero for $\mu_{B}<M_{\pi}$. Then, the $\mathcal{O}\left(p^{4}\right)$ corrections appear only for $\mu_{B}>M_{\pi}$, where the $U(1)_{B}$ is spontaneously broken. It should be noticed that two 

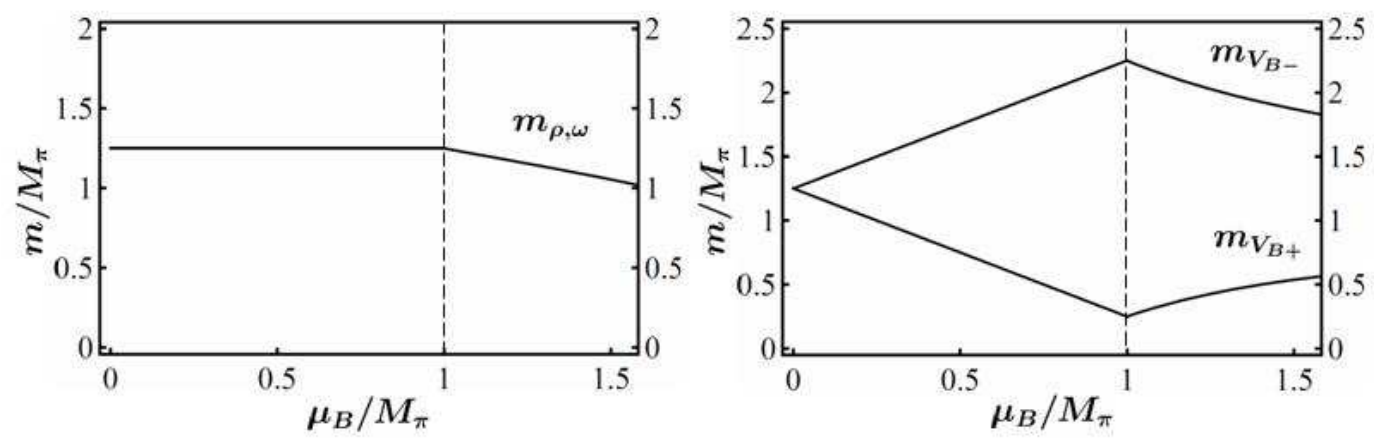

FIG. 4: $\mu_{B}$-dependences of the "vector" boson masses. The curve on the left figure is for the degenerate $\rho$ and $\omega$ mesons and those on the right figure are for baryonic and anti-baryonic "vector" bosons. We use $M_{V} / M_{\pi}=5 / 4, C_{1}=0, C_{2}=1$ and $g=1$ to make the curves.

coefficients $C_{1}$ and $C_{2}$ are not determined by the symmetry structure. To study the effect of $\mathcal{O}\left(p^{4}\right)$ corrections we refer to the mass spectra obtained by the lattice simulation in Ref. [7], which shows that the masses of $\rho$ and $\omega$ mesons are degenerate, and that both of them are stable against the change of the chemical potential for $\mu_{B}<M_{\pi}$ and decrease for $\mu_{B}>M_{\pi}$. From Eqs. (4.6) and (4.7) the degeneracy of $m_{\rho}$ and $m_{\omega}$ is realized for $C_{1}=0$, and both $m_{\rho}$ and $m_{\omega}$ decrease for $C_{2}>0$. From Eq. (4.5) together with $X^{4} X^{4}=1$, the choice of $C_{1}=0$ and $C_{2}>0$ implies that the $\mathcal{O}\left(p^{4}\right)$ terms provide a negative contribution to all the "vector" boson masses equally, and that $V_{B_{+}}$and $V_{B_{-}}$do not mix with each other, even though the baryon number $U(1)_{B}$ symmetry is spontaneously broken. On the other hand, the choice of $C_{1}=0$ and $C_{2}<0$ implies that the $\mathcal{O}\left(p^{4}\right)$ terms provide a positive contribution to all the "vector" boson masses equally (see FIG. 3). The effect of nonzero $C_{1}$ produces the mass difference between $m_{\rho}$ and $m_{\omega}$, and this difference is linked to the mixing strength between $V_{B_{+}}$and $V_{B_{-}}$as in Eq. (4.9). This relation is obtained from the symmetry breaking pattern and the assumption that all the bosons other than $\pi$ and $V$ are heavy enough to be neglected in the Lagrangian. \#5 Thus, a violation of Eq. (4.9), when only $\pi$ and $V$ are light degrees of freedom, may signal a new phase transition.

We consider the case with $M_{V} / M_{\pi}=5 / 4$, i.e., relatively heavy $\pi \mathrm{s}$, which corresponds to the one in the lattice analysis [7]. As an example, we plot the $\mu_{B}$-dependence of the "vector"

\#5 We expect that the contribution of higher order terms such as $\mathcal{O}\left(p^{6}\right)$ is small enough. 

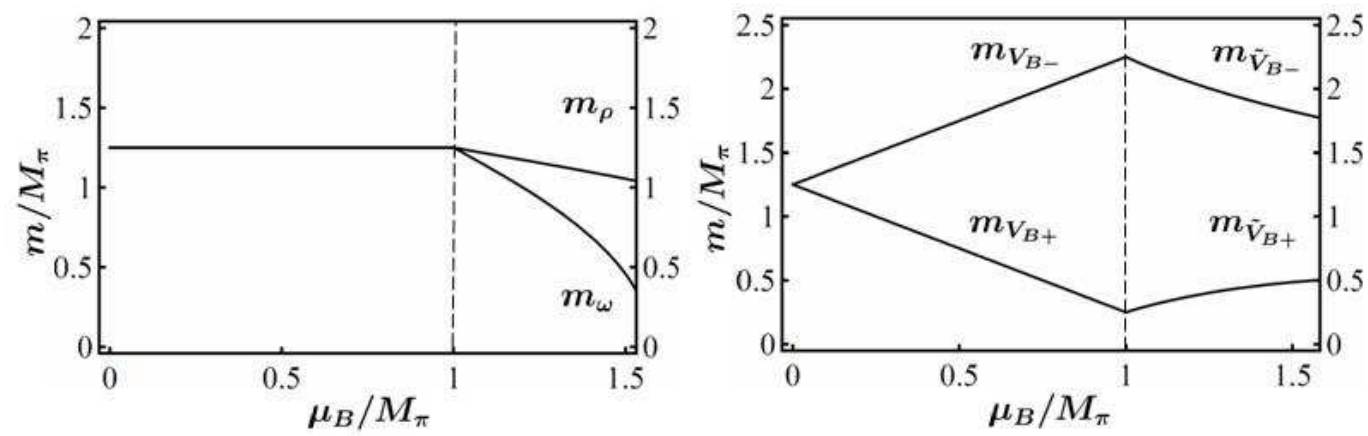

FIG. 5: $\mu_{B}$-dependences of the "vector" boson masses. The curves on the left figure are for the $\rho$ and $\omega$ mesons and those on the right figure are for baryonic and anti-baryonic "vector" bosons. We use $M_{V} / M_{\pi}=5 / 4, C_{1}=1, C_{2}=2$ and $g=1$ to make the curves.

boson masses for $C_{1}=0$ and $C_{2}=1$ together with $M_{V} / M_{\pi}=5 / 4$ and $g=1$ in FIG. 4. We choose the value $C_{2}=1$ to reproduce the decreasing masses of $\rho$ and $\omega$ mesons in the lattice data [7] for $\mu_{B}>M_{\pi}$. We obtain the result that $m_{V_{B+}}$ increases and $m_{V_{B-}}$ decreases with increasing $\mu_{B}$ for $\mu_{B}>M_{\pi}$. The lattice analysis [7] shows that $m_{V_{B+}}$ is almost stable against the change of $\mu_{B}$, which does not agree with the result of the present analysis. Though any clear signal for $m_{V_{B-}}$ is not observed.

We investigate the effect of $C_{1}$ term to $m_{V_{B+}}$ and $m_{V_{B-}}$ in Eq. (4.8). This term causes a mixing between $V_{B_{+}}$and $V_{B_{-}}$, and makes $m_{V_{B+}}\left(m_{V_{B_{-}}}\right)$smaller (larger). At the same time, the $C_{1}$ term produces the mass difference between the $\rho$ and $\omega$ mesons (see Eq. (4.9)): The positive $C_{1}$ gives the positive correction to $m_{\rho}$ and the negative one to $m_{\omega}$, and the negative $C_{1}$ gives the negative correction to $m_{\rho}$ and the positive one to $m_{\omega}$. As an example, we plot the $\mu_{B}$-dependence of the "vector" boson masses for $C_{1}=1$ and $C_{2}=2$ together with $M_{V} / M_{\pi}=5 / 4$ and $g=1$ in FIG. 5. We set $C_{2}-C_{1}=1$ to reproduce the decreasing mass of $\rho$ meson in the lattice data [7], and $C_{1}=1$ to produce $10 \%$ decreasing of $m_{V_{B+}}$ at $\mu_{B} / M_{\pi}=1.5$. Left panel of FIG. 5 shows that the splitting between $m_{\rho}$ and $m_{\omega}$ is large: $m_{\omega}$ is about half of $m_{\rho}$ at $\mu_{B} / M_{\pi}=1.5$. Thus, our model cannot simultaneously reproduce the $\mu_{B}$-dependences of $m_{\rho}, m_{\omega}$ and $m_{V_{B+}}$ in the lattice data [7].

For relatively heavy $\pi \mathrm{s}$, we expect that the masses of "axial-vector" bosons are smaller than $M_{V}+M_{\pi}$ which is the largest value of "vector" boson masses in the present analysis, so that we cannot neglect the "axial-vector" bosons. When we include the "axial-vector" 
meson $A$, as well as the baryonic and anti-baryonic "axial-vector" bosons, $A_{B_{+}}$and $A_{B_{-}}$ (see Table III), the $A$ will mix with baryonic and anti-baryonic "vector" bosons, $V_{B_{+}}$and $V_{B_{-}}$, and $A_{B_{+}}$and $A_{B_{-}}$will mix with $\omega$ meson for $\mu_{B}>M_{\pi}$. The effect of mixing among $A$, $V_{B_{+}}$and $V_{B_{-}}$will make $m_{V_{B+}}$ lighter since $m_{V_{B+}}$ is the smallest among three at $\mu_{B}=M_{\pi}$. As a result, the $m_{V_{B+}}$ becomes more stable than that shown in FIG. 4, and the lattice data will be reproduced. This strongly suggests that there is a large mixing among $A, V_{B_{+}}$and $V_{B_{-}}$for heavy $\pi \mathrm{s}$ such as the one adopted in the lattice analysis [7]. This indicates that the mass of isovector meson with $J^{P}=1^{+}$shown in FIG. 2 of Ref. [7] is nothing but $m_{V_{B+}}$ observed through the large mixing. On the other hand, the mixing among $A_{B_{+}}, A_{B_{-}}$and $\omega$ will generally break the degeneracy between the $\rho$ and $\omega$ mesons as shown in e.g. Ref. [8], which does not seem to agree with the lattice result. Then, the mixing expected to small enough to reproduce the lattice result.

\begin{tabular}{ccccc}
\hline Field & Generator & $I$ & $B$ & $J^{P}$ \\
\hline$A^{1,2,3}$ & $X^{1,2,3}$ & 1 & 0 & $1^{+}$ \\
$A_{B_{+}}=\left(A^{5}-i A^{4}\right) / \sqrt{2}$ & $\left(X^{5}-i X^{4}\right) / \sqrt{2}$ & 0 & +1 & $1^{-}$ \\
$A_{B_{-}}=\left(A^{5}+i A^{4}\right) / \sqrt{2}$ & $\left(X^{5}+i X^{4}\right) / \sqrt{2}$ & 0 & -1 & $1^{-}$ \\
\hline
\end{tabular}

TABLE III: Summary of the "axial-vector" fields

\section{SUMMARY AND DISCUSSIONS}

In this paper, we constructed a low energy effective Lagrangian for the two-color QCD including the "vector" bosons (mesons with $J^{P}=1^{-}$and diquark baryons with $J^{P}=1^{+}$) in addition to the pseudo Nambu-Goldstone bosons (mesons with $J^{P}=0^{-}$and baryons with $\left.J^{P}=0^{+}\right)$based on the chiral symmetry breaking pattern of $S U\left(2 N_{f}\right) \rightarrow S p\left(2 N_{f}\right)$ in the framework of the hidden local symmetry (HLS). The "vector" bosons were introduced as the gauge bosons of the $S p\left(2 N_{f}\right)$ HLS. In the HLS formalism, the "vector" bosons and the external chiral gauge bosons are included independently, so that we can naturally incorporate the chemical potential $\mu_{B}$ as the vacuum expectation value (VEV) of the external gauge boson for baryon number.

We studied the vacuum structure of the model in the case of $N_{f}=2$ introducing the effects of current quark masses and the baryon chemical potential into the leading order 
Lagrangian. We found that the time component of " $\omega$ " meson has a VEV for any $\mu_{B}$, as in the ordinary three-color QCD. As a result, the phase structure is the same as the one determined by including only the pseudo-NG bosons [3]: For $\mu_{B}>M_{\pi}$, the baryonic pion $\left(J^{P}=0^{+}\right.$state) condenses, which causes the spontaneous breaking of the baryon number symmetry, $U(1)_{B}$.

We investigated the $\mu_{B}$-dependences of the "vector" boson masses and found that the mass of anti-baryon with $J^{P}=1^{+}\left(V_{B_{-}}\right)$increases for $\mu_{B}<M_{\pi}$ and turns to decrease for $\mu_{B}>M_{\pi}$. The mass of baryon with $J^{P}=1^{+}\left(V_{B_{+}}\right)$shows the opposite behavior. These behaviors of the baryons signal the phase transition of $U(1)_{B}$ breaking in two-color QCD at finite density.

At the leading order the vector mesons with $J^{P}=1^{-}$( $\rho$ and $\omega$ mesons) do not change their masses at all. Furthermore, $V_{B_{-}}$does not mix with $V_{B_{+}}$, even though the baryon number $U(1)_{B}$ symmetry is spontaneously broken for $\mu_{B}>M_{\pi}$.

We studied the effects of higher order terms and obtained the corrections to the masses of the "vector" bosons. We assumed that the vacuum structure is not changed, which left only two free parameters $C_{1}$ and $C_{2}$ : The positive (negative) $C_{2}$ provides a negative (positive) contribution to all the "vector" boson masses equally. On the other hand, the effect of nonzero $C_{1}$ produces the mass difference between $m_{\rho}$ and $m_{\omega}$, and this difference is linked to the mixing strength between $V_{B_{+}}$and $V_{B_{-}}$. This relation is obtained from the symmetry breaking pattern and the assumption that all the bosons other than $\pi$ and $V$ are heavy enough to be neglected in the Lagrangian. Thus, a violation of this relation (Eq. (4.9)) may signal a new phase transition.

Comparison with the lattice data in Ref. [7] strongly suggests that there is a large mixing among $A, V_{B_{+}}$and $V_{B_{-}}$for heavy $\pi$ s such as the one adopted in Ref. [7]. This indicates that the mass of isovector meson with $J^{P}=1^{+}$shown in FIG. 2 of Ref. [7] is nothing but $m_{V_{B}}$ observed through the large mixing.

We make a comment on the analysis done in Ref. [8]. When the "axial-vector" bosons are taken to be heavy in their model and integrated out, the model becomes identical to the HLS model with the parameter choice $C_{1}=C_{2}=2\left(M_{S} / M_{X}\right)^{4} / g^{2}$, where $M_{S}$ and $M_{X}$ express the masses of "vector" bosons and "axial-vector" bosons in their model. As can be seen easily from Eq. (4.6), the choice $C_{1}=C_{2}$ implies that the $\rho$ meson mass $m_{\rho}$ is stable against the density as shown in Ref. [8]. 
The present analysis is valid when the "axial-vector" bosons (mesons with $J^{P}=1^{+}$and baryons with $J^{P}=1^{-}$) are heavy. When the "axial-vector" bosons are light, we need to include these states. This can be done in the framework of the generalized HLS [10, 19]. Using this formalism, we may investigate the phase structure in the range of $\mu_{B}$ wider than that studied in the present analysis. We hope to obtain a clue to understand the real-life QCD with three colors at finite baryon density through these analyses.

\section{Acknowledgments}

This work is supported in part by Global COE Program "Quest for Fundamental Principles in the Universe" of Nagoya University (G07). The work of M.H. is supported in part by the JSPS Grant-in-Aid for Scientific Research (c) 20540262 and Grant-in-Aid for Scientific Research on Innovative Areas (No. 2104) "Quest on New Hadrons with Variety of Flavors" from MEXT. The work of C.N. is supported in part by the Mitsubishi Foundation.

\section{Appendix A: QCD Lagrangian with external source fields}

In this appendix, we give the QCD Lagrangian with the external source fields.

We start with the ordinary QCD Lagrangian with $N_{f}$ massless quarks:

$$
\mathcal{L}_{\mathrm{QCD}}^{0}=-\frac{1}{2} \operatorname{tr}\left[G^{\nu \rho} G_{\nu \rho}\right]+\bar{\psi} \gamma^{\nu} D_{\nu} \psi
$$

where

$$
\begin{aligned}
D_{\nu} \psi & =\left(\partial_{\nu}-i g_{s} G_{\nu}\right) \psi, \\
G_{\nu \rho} & =\partial_{\nu} G_{\rho}-\partial_{\rho} G_{\nu}-i g_{s}\left[G_{\nu}, G_{\rho}\right],
\end{aligned}
$$

with $G_{\nu}$ and $g_{s}$ being the gluon field matrix and the gauge coupling constant. Note that the gluon field matrix is expressed as $G_{\nu}=G_{\nu}^{a} \frac{\tau_{a}}{2}$ where $\tau_{a}$ is the Pauli matrix of $S U(2)_{\text {color }}$ defined as

$$
\tau_{1}=\left(\begin{array}{ll}
0 & 1 \\
1 & 0
\end{array}\right), \tau_{2}=\left(\begin{array}{cc}
0 & -i \\
i & 0
\end{array}\right), \tau_{3}=\left(\begin{array}{cc}
1 & 0 \\
0 & -1
\end{array}\right)
$$


We include external scalar and pseudoscalar source fields $\mathcal{S}$ and $\mathcal{P}$, as well as scalar and pseudoscalar diquark source fields $\mathcal{Q}$ and $\mathcal{R} \# 6$ as

$$
\mathcal{L}_{\text {ext-scalar }}=\bar{\psi}(\mathcal{S}+i \mathcal{P}) \psi+\frac{1}{2}\left[\psi^{T} C\left(\gamma_{5} \mathcal{Q}+i \mathcal{R}\right) i \tau_{2} \psi+(\text { h.c })\right]
$$

The vector and axial-vector external gauge fields $\mathcal{V}^{\mu}$ and $\mathcal{A}^{\mu}$ as well as diquark external gauge fields carrying $J^{P}=1^{+}$and $1^{-}\left(\mathcal{B}^{\mu}\right.$ and $\left.\mathcal{D}^{\mu}\right)$ are included as

$$
\mathcal{L}_{\text {ext-vector }}=\bar{\psi} \gamma_{\mu}\left(\mathcal{V}^{\mu}-\gamma_{5} \mathcal{A}^{\mu}\right) \psi+\frac{1}{2}\left[\psi^{T} C \gamma_{\mu}\left(\mathcal{B}^{\mu}-\gamma_{5} \mathcal{D}^{\mu}\right) i \tau_{2} \psi+(\text { h.c })\right]
$$

These external fields satisfy the following conditions:

$$
\begin{aligned}
& \mathcal{S}^{\dagger}=\mathcal{S}, \quad \mathcal{P}^{\dagger}=\mathcal{P}, \quad\left(\mathcal{V}^{\mu}\right)^{\dagger}=\mathcal{V}^{\mu}, \quad\left(\mathcal{A}^{\mu}\right)^{\dagger}=\mathcal{A}^{\mu}, \\
& \mathcal{Q}^{T}=-\mathcal{Q}, \quad \mathcal{R}^{T}=-\mathcal{R}, \quad\left(\mathcal{B}^{\mu}\right)^{T}=\mathcal{B}^{\mu}, \quad\left(\mathcal{D}^{\mu}\right)^{T}=-\mathcal{D}^{\mu}
\end{aligned}
$$

Now, the total Lagrangian is given by

$$
\mathcal{L}_{\mathrm{QCD}}=\mathcal{L}_{\mathrm{QCD}}^{0}+\mathcal{L}_{\text {ext-scalar }}+\mathcal{L}_{\text {ext-vector }}
$$

It is convenient to introduce two-component spinors $q_{L}$ and $q_{R}$ in such a way that the four-component spinor $\psi$ is expressed as $\psi^{i}=\left(\begin{array}{c}q_{L}^{i} \\ q_{R}^{i}\end{array}\right)$, where $i$ denotes the flavor index. Then, the kinetic term of quarks is rewritten as

$$
\int d^{4} x i \bar{\psi}_{i} \gamma_{\nu} D^{\nu} \psi^{i}=\int d^{4} x\left(i q_{L, i}^{\dagger} \sigma_{\nu} D^{\nu} q_{L}^{i}+i q_{R, i}^{\dagger} \bar{\sigma}_{\nu} D^{\nu} q_{R}^{i}\right)
$$

where we use the following form of the gamma matrices:

$$
\gamma_{\nu}=\left(\begin{array}{cc}
\mathbf{0} & \bar{\sigma}_{\nu} \\
\sigma_{\nu} & \mathbf{0}
\end{array}\right) \equiv\left(\begin{array}{cc}
\mathbf{0} & \left(\mathbf{1}, \sigma_{j}\right) \\
\left(\mathbf{1},-\sigma_{j}\right) & \mathbf{0}
\end{array}\right)
$$

From the form given in Eq.(A.8) the existence of the $S U\left(2 N_{f}\right)$ flavor symmetry is seen as follows: Since the fundamental representation of $S U(2)_{\text {color }}$ as well as that of $S U(2)_{\text {spin }}$ is the pseudreal representation, a combination of $\sigma_{2} \tau_{2} q_{R}^{*}$ has the same transformation property

$\# 6 \mathcal{Q}$ and $\mathcal{R}$ are color-singlet states in two-color QCD. This can be seen from the existence of $i \tau_{2}=\epsilon$ and the charge-conjugation matrix $C \equiv i \gamma_{2} \gamma_{0}$ in Eq. A.4. 
as $q_{L}$ under the $S U(2)_{\text {color }}$ symmetry as well as under the Lorentz symmetry. Then, by introducing the field $\Psi$ as

$$
\Psi=\left(\begin{array}{c}
q_{L}^{1} \\
q_{L}^{2} \\
\vdots \\
q_{L}^{N_{f}} \\
\sigma_{2} \tau_{2} q_{R, 1}^{*} \\
\sigma_{2} \tau_{2} q_{R, 2}^{*} \\
\vdots \\
\sigma_{2} \tau_{2} q_{R, N_{f}}^{*}
\end{array}\right)
$$

the kinetic term in Eq.(A.8) is rewritten as

$$
\int d^{4} x i \bar{\psi} \gamma_{\nu} D^{\nu} \psi=\int d^{4} x i \Psi^{\dagger} \sigma_{\nu} D^{\nu} \Psi
$$

This is invariant under the $S U\left(2 N_{f}\right)$ transformation of $\Psi$ given as

$$
\Psi \rightarrow g \Psi, \quad\left(g \in S U\left(2 N_{f}\right)\right)
$$

Similarly, using the field $\Psi$, we rewrite $\mathcal{L}_{\text {ext-scalar }}$ and $\mathcal{L}_{\text {ext-vector in Eqs. (A.4) and (A.5) as }}$

$$
\left.\mathcal{L}_{\text {ext-scalar }}=\frac{1}{2} \Psi^{T} \sigma_{2} \tau_{2} \chi \Psi+\text { (h.c }\right), \quad \mathcal{L}_{\text {ext-vector }}=\Psi^{\dagger} \sigma_{\mu} G^{\mu} \Psi
$$

where $\chi$ and $G^{\mu}$ are external fields of $2 N_{f} \times 2 N_{f}$ matrices defined as

$$
\begin{aligned}
\chi \equiv\left(\begin{array}{cc}
\mathcal{Q}-i \mathcal{R} & -(\mathcal{S}-i \mathcal{P})^{T} \\
\mathcal{S}-i \mathcal{R} & (\mathcal{Q}+i \mathcal{R})^{\dagger}
\end{array}\right) \\
G^{\mu} \equiv\left(\begin{array}{cc}
\mathcal{V}^{\mu}+\mathcal{A}^{\mu} & \left(\mathcal{B}^{\mu}+\mathcal{D}^{\mu}\right)^{\dagger} \\
\mathcal{B}^{\mu}+\mathcal{D}^{\mu} & -\left(\mathcal{V}^{\mu}-\mathcal{A}^{\mu}\right)^{T}
\end{array}\right)
\end{aligned}
$$

Transformation properties of the external fields under $S U\left(2 N_{f}\right)$ are given by

$$
G_{\mu} \rightarrow g G_{\mu} g^{\dagger}+i g\left(\partial_{\mu} g^{\dagger}\right), \quad \chi \rightarrow g^{*} \chi g^{\dagger}
$$

\section{Appendix B: Explicit realization of the $S U(4)$ generators}

In this appendix, we show the explicit representation of the generators of $S U(4)$. We consider the form of the generators following Ref. [8] for convenience. They can be represented 
as

$$
S^{\alpha}=\frac{1}{2 \sqrt{2}}\left(\begin{array}{cc}
\boldsymbol{A} & \boldsymbol{B} \\
\boldsymbol{B}^{\dagger} & -\boldsymbol{A}^{T}
\end{array}\right), \quad X^{a}=\frac{1}{2 \sqrt{2}}\left(\begin{array}{cc}
\boldsymbol{C} & \boldsymbol{D} \\
\boldsymbol{D}^{\dagger} & \boldsymbol{C}^{T}
\end{array}\right),
$$

where $\boldsymbol{A}$ is hermitian, $\boldsymbol{C}$ is hermitian and traceless, $\boldsymbol{B}^{T}=\boldsymbol{B}$ and $\boldsymbol{D}^{T}=-\boldsymbol{D}$. The $\{S\}$ are also $S p(4)$ generators since they obey Eq. (2.1). We define

$$
S^{\alpha}=\frac{1}{2 \sqrt{2}}\left(\begin{array}{cc}
\tau^{\alpha} & \mathbf{0} \\
\mathbf{0} & -\left(\tau^{\alpha}\right)^{T}
\end{array}\right), \quad(\alpha=1,2,3,4) .
$$

For $\alpha=1,2,3$, we have the standard Pauli matrices, while for $\alpha=4$ we define $\tau^{4}=\mathbf{1}$. These are simply the generators for $S U(2) \times U(1)$. For $\alpha=5, \ldots, 10$

$$
S^{\alpha}=\frac{1}{2 \sqrt{2}}\left(\begin{array}{cc}
\mathbf{0} & B^{\alpha} \\
\left(B^{\alpha}\right)^{\dagger} & \mathbf{0}
\end{array}\right), \quad(\alpha=5, \ldots, 10),
$$

and

$$
B^{5}=\mathbf{1}_{2}, B^{6}=i \mathbf{1}_{2}, B^{7}=\tau^{3}, B^{8}=i \tau^{3}, B^{9}=\tau^{1}, B^{10}=i \tau^{1} .
$$

The five broken generators $\{X\}$ are

$$
X^{a}=\frac{1}{2 \sqrt{2}}\left(\begin{array}{cc}
\tau^{a} & \mathbf{0} \\
\mathbf{0} & \left(\tau^{a}\right)^{T}
\end{array}\right), \quad(a=1,2,3),
$$

where $\tau^{a}$ are the standard Pauli matrices. For $a=4,5$

$$
X^{a}=\frac{1}{2 \sqrt{2}}\left(\begin{array}{cc}
\mathbf{0} & D^{a} \\
\left(D^{a}\right)^{\dagger} & \mathbf{0}
\end{array}\right), \quad(a=4,5),
$$

and

$$
D^{4}=\tau^{2}, D^{5}=i \tau^{2}
$$

The generators are normalized as follows:

$$
\operatorname{tr}\left(S^{\alpha} S^{\beta}\right)=\frac{1}{2} \delta^{\alpha \beta}, \quad \operatorname{tr}\left(X^{a} X^{b}\right)=\frac{1}{2} \delta^{a b}, \quad \operatorname{tr}\left(S^{\alpha} X^{a}\right)=0 .
$$

\section{Appendix C: $\mathcal{O}\left(p^{4}\right)$ HLS Lagrangian}

In this appendix, we present a complete list of the $\mathcal{O}\left(p^{4}\right)$ HLS Lagrangian for general $N_{f}$ and $N_{C}=2$, following Refs. [11, 17]. For the construction, we need the building blocks

$$
\hat{\mathcal{V}}_{\mu \nu}=\frac{1}{2}\left[\xi_{R} \bar{\Sigma} G_{\mu \nu}^{T} \bar{\Sigma} \xi_{R}^{\dagger}+\xi_{L} G_{\mu \nu} \xi_{L}^{\dagger}\right], \quad \hat{\mathcal{A}}_{\mu \nu}=\frac{1}{2}\left[\xi_{R} \bar{\Sigma} G_{\mu \nu}^{T} \bar{\Sigma} \xi_{R}^{\dagger}-\xi_{L} G_{\mu \nu} \xi_{L}^{\dagger}\right],
$$


where $G_{\mu \nu}$ is the filed strength of the external chiral gauge fields defined as

$$
G_{\mu \nu} \equiv \partial_{\mu} G_{\nu}-\partial_{\nu} G_{\mu}-i\left[G_{\mu}, G_{\nu}\right]
$$

From Eq. (C.1) together with other building blocks in TABLE【, a complete list of the $\mathcal{O}\left(p^{4}\right)$ Lagrangian invariant under the $C$ and $P$ transformation is constructed as

$$
\mathcal{L}_{(4)}=\mathcal{L}_{(4) y}+\mathcal{L}_{(4) w}+\mathcal{L}_{(4) z}
$$

where

$$
\begin{aligned}
& \mathcal{L}_{(4) y}=y_{1} \operatorname{tr}\left[\hat{\alpha}_{\perp \mu} \hat{\alpha}_{\perp}^{\mu} \hat{\alpha}_{\perp \nu} \hat{\alpha}_{\perp}^{\nu}\right]+y_{2} \operatorname{tr}\left[\hat{\alpha}_{\perp \mu} \hat{\alpha}_{\perp \nu} \hat{\alpha}_{\perp}^{\mu} \hat{\alpha}_{\perp}^{\nu}\right] \\
& +y_{3} \operatorname{tr}\left[\hat{\alpha}_{\| \mu} \hat{\alpha}_{\|}^{\mu} \hat{\alpha}_{\| \nu} \hat{\alpha}_{\|}^{\nu}\right]+y_{4} \operatorname{tr}\left[\hat{\alpha}_{\| \mu} \hat{\alpha}_{\| \nu} \hat{\alpha}_{\|}^{\mu} \hat{\alpha}_{\|}^{\nu}\right] \\
& +y_{5} \operatorname{tr}\left[\hat{\alpha}_{\perp \mu} \hat{\alpha}_{\perp}^{\mu} \hat{\alpha}_{\| \nu} \hat{\alpha}_{\|}^{\nu}\right]+y_{6} \operatorname{tr}\left[\hat{\alpha}_{\perp \mu} \hat{\alpha}_{\perp \nu} \hat{\alpha}_{\|}^{\mu} \hat{\alpha}_{\|}^{\nu}\right]+y_{7} \operatorname{tr}\left[\hat{\alpha}_{\perp \mu} \hat{\alpha}_{\perp \nu} \hat{\alpha}_{\|}^{\nu} \hat{\alpha}_{\|}^{\mu}\right] \\
& +y_{8}\left\{\operatorname{tr}\left[\hat{\alpha}_{\perp \mu} \hat{\alpha}_{\|}^{\mu} \hat{\alpha}_{\perp \nu} \hat{\alpha}_{\|}^{\nu}\right]+\operatorname{tr}\left[\hat{\alpha}_{\perp \mu} \hat{\alpha}_{\| \nu} \hat{\alpha}_{\perp}^{\nu} \hat{\alpha}_{\|}^{\mu}\right]\right\}+y_{9} \operatorname{tr}\left[\hat{\alpha}_{\perp \mu} \hat{\alpha}_{\| \nu} \hat{\alpha}_{\perp}^{\mu} \hat{\alpha}_{\|}^{\nu}\right] \\
& +y_{10}\left(\operatorname{tr}\left[\hat{\alpha}_{\perp \mu} \hat{\alpha}_{\perp}^{\mu}\right]\right)^{2}+y_{11} \operatorname{tr}\left[\hat{\alpha}_{\perp \mu} \hat{\alpha}_{\perp \nu}\right] \operatorname{tr}\left[\hat{\alpha}_{\perp}^{\mu} \hat{\alpha}_{\perp}^{\nu}\right] \\
& +y_{12}\left(\operatorname{tr}\left[\hat{\alpha}_{\| \mu} \hat{\alpha}_{\|}^{\mu}\right]\right)^{2}+y_{13} \operatorname{tr}\left[\hat{\alpha}_{\| \mu} \hat{\alpha}_{\| \nu}\right] \operatorname{tr}\left[\hat{\alpha}_{\|}^{\mu} \hat{\alpha}_{\|}^{\nu}\right] \\
& +y_{14} \operatorname{tr}\left[\hat{\alpha}_{\perp \mu} \hat{\alpha}_{\perp}^{\mu}\right] \operatorname{tr}\left[\hat{\alpha}_{\| \nu} \hat{\alpha}_{\|}^{\nu}\right]+y_{15} \operatorname{tr}\left[\hat{\alpha}_{\perp \mu} \hat{\alpha}_{\perp \nu}\right] \operatorname{tr}\left[\hat{\alpha}_{\|}^{\mu} \hat{\alpha}_{\|}^{\nu}\right] \\
& \mathcal{L}_{(4) w}=w_{1} \frac{f_{\chi}^{2}}{f_{\pi}^{2}} \operatorname{tr}\left[\hat{\alpha}_{\perp \mu} \hat{\alpha}_{\perp}^{\mu}\left(\hat{\chi}+\hat{\chi}^{\dagger}\right)\right]+w_{2} \frac{f_{\chi}^{2}}{f_{\pi}^{2}} \operatorname{tr}\left[\hat{\alpha}_{\perp \mu} \hat{\alpha}_{\perp}^{\mu}\right] \operatorname{tr}\left[\hat{\chi}+\hat{\chi}^{\dagger}\right] \\
& +w_{3} \frac{f_{\chi}^{2}}{f_{\pi}^{2}} \operatorname{tr}\left[\hat{\alpha}_{\| \mu} \hat{\alpha}_{\|}^{\mu}\left(\hat{\chi}+\hat{\chi}^{\dagger}\right)\right]+w_{4} \frac{f_{\chi}^{2}}{f_{\pi}^{2}} \operatorname{tr}\left[\hat{\alpha}_{\| \mu} \hat{\alpha}_{\|}^{\mu}\right] \operatorname{tr}\left[\hat{\chi}+\hat{\chi}^{\dagger}\right] \\
& +w_{5} \frac{f_{\chi}^{2}}{f_{\pi}^{2}} \operatorname{tr}\left[\left(\hat{\alpha}_{\|}^{\mu} \hat{\alpha}_{\perp \mu}-\hat{\alpha}_{\perp \mu} \hat{\alpha}_{\|}^{\mu}\right)\left(\hat{\chi}-\hat{\chi}^{\dagger}\right)\right] \\
& +w_{6} \frac{f_{\chi}^{2}}{f_{\pi}^{2}} \operatorname{tr}\left[\left(\hat{\chi}+\hat{\chi}^{\dagger}\right)^{2}\right]+w_{7} \frac{f_{\chi}^{2}}{f_{\pi}^{2}}\left(\operatorname{tr}\left[\hat{\chi}+\hat{\chi}^{\dagger}\right]\right)^{2} \\
& +w_{8} \frac{f_{\chi}^{2}}{f_{\pi}^{2}} \operatorname{tr}\left[\left(\hat{\chi}-\hat{\chi}^{\dagger}\right)^{2}\right]+w_{9} \frac{f_{\chi}^{2}}{f_{\pi}^{2}}\left(\operatorname{tr}\left[\hat{\chi}-\hat{\chi}^{\dagger}\right]\right)^{2}, \\
& \mathcal{L}_{(4) z}=z_{1} \operatorname{tr}\left[\hat{\mathcal{V}}_{\mu \nu} \hat{\mathcal{V}}^{\mu \nu}\right]+z_{2} \operatorname{tr}\left[\hat{\mathcal{A}}_{\mu \nu} \hat{\mathcal{A}}^{\mu \nu}\right]+z_{3} \operatorname{tr}\left[\hat{\mathcal{V}}_{\mu \nu} V^{\mu \nu}\right] \\
& +i z_{4} \operatorname{tr}\left[V_{\mu \nu} \hat{\alpha}_{\perp}^{\mu} \hat{\alpha}_{\perp}^{\nu}\right]+i z_{5} \operatorname{tr}\left[V_{\mu \nu} \hat{\alpha}_{\|}^{\mu} \hat{\alpha}_{\|}^{\nu}\right] \\
& +i z_{6} \operatorname{tr}\left[\hat{\mathcal{V}}_{\mu \nu} \hat{\alpha}_{\perp}^{\mu} \hat{\alpha}_{\perp}^{\nu}\right]+i z_{7} \operatorname{tr}\left[\hat{\mathcal{V}}_{\mu \nu} \hat{\alpha}_{\|}^{\mu} \hat{\alpha}_{\|}^{\nu}\right]-i z_{8} \operatorname{tr}\left[\hat{\mathcal{A}}_{\mu \nu}\left(\hat{\alpha}_{\perp}^{\mu} \hat{\alpha}_{\|}^{\nu}+\hat{\alpha}_{\|}^{\mu} \hat{\alpha}_{\perp}^{\nu}\right)\right] .
\end{aligned}
$$

[1] See e.g. S. Muroya, A. Nakamura, C. Nonaka and T. Takaishi, Prog. Theor. Phys. 110, 615 (2003). 
[2] J. B. Kogut, M. A. Stephanov and D. Toublan, Phys. Lett. B 464, 183 (1999); K. Splittorff, D. T. Son and M. A. Stephanov, Phys. Rev. D 64, 016003 (2001); K. Splittorff, D. Toublan and J. J. M. Verbaarschot, Nucl. Phys. B 620, 290 (2002); J. Wirstam, J. T. Lenaghan and K. Splittorff, Phys. Rev. D 67, 034021 (2003); C. Ratti and W. Weise, Phys. Rev. D 70, 054013 (2004); T. Kanazawa, T. Wettig and N. Yamamoto, JHEP 0908, 003 (2009); T. Brauner, K. Fukushima and Y. Hidaka, Phys. Rev. D 80, 074035 (2009).

[3] J. B. Kogut, M. A. Stephanov, D. Toublan, J. J. M. Verbaarschot and A. Zhitnitsky, Nucl. Phys. B 582, 477 (2000).

[4] S. Hands, I. Montvay, S. Morrison, M. Oevers, L. Scorzato and J. Skullerud, Eur. Phys. J. C 17, 285 (2000); S. Hands, I. Montvay, L. Scorzato and J. Skullerud, Eur. Phys. J. C 22, 451 (2001).

[5] S. Hands, J. B. Kogut, M. P. Lombardo and S. E. Morrison, Nucl. Phys. B 558, 327 (1999); J. B. Kogut, D. K. Sinclair, S. J. Hands and S. E. Morrison, Phys. Rev. D 64, 094505 (2001); J. B. Kogut, D. Toublan and D. K. Sinclair, Nucl. Phys. B 642, 181 (2002); J. B. Kogut, D. Toublan and D. K. Sinclair, Phys. Rev. D 68, 054507 (2003); Y. Nishida, K. Fukushima and T. Hatsuda, Phys. Rept. 398, 281 (2004).

[6] S. Muroya, A. Nakamura and C. Nonaka, Phys. Lett. B 551, 305 (2003).

[7] S. Hands, P. Sitch and J. I. Skullerud, Phys. Lett. B 662, 405 (2008).

[8] J. T. Lenaghan, F. Sannino and K. Splittorff, Phys. Rev. D 65, 054002 (2002).

[9] M. Bando, T. Kugo, S. Uehara, K. Yamawaki and T. Yanagida, Phys. Rev. Lett. 54, 1215 (1985).

[10] M. Bando, T. Kugo and K. Yamawaki, Phys. Rept. 164, 217 (1988).

[11] M. Harada and K. Yamawaki, Phys. Rept. 381, 1 (2003).

[12] C. G. Callan, S. R. Coleman, J. Wess and B. Zumino, Phys. Rev. 177, 2247 (1969); S. R. Coleman, J. Wess and B. Zumino, Phys. Rev. 177, 2239 (1969); G. Ecker, J. Gasser, H. Leutwyler, A. Pich and E. de Rafael, Phys. Lett. B 223, 425 (1989).

[13] J. Gasser and H. Leutwyler, Annals Phys. 158, 142 (1984); G. Ecker, J. Gasser, A. Pich and E. de Rafael, Nucl. Phys. B 321, 311 (1989); G. Ecker, J. Gasser, H. Leutwyler, A. Pich and E. de Rafael, Phys. Lett. B 223, 425 (1989).

[14] J. Wess and B. Zumino, Phys. Rev. 163, 1727 (1967); J. S. Schwinger, Phys. Lett. B 24, 473 (1967); S. Gasiorowicz and D. A. Geffen, Rev. Mod. Phys. 41, 531 (1969); O. Kaymak- 
calan, S. Rajeev and J. Schechter, Phys. Rev. D 30, 594 (1984); J. Schechter, Phys. Rev. D 34, 868 (1986); M. F. L. Golterman and N. D. Hari Dass, Nucl. Phys. B 277, 739 (1986); U. G. Meissner, Phys. Rept. 161, 213 (1988); U. G. Meissner and I. Zahed, Z. Phys. A 327, 5 (1987).

[15] H. Georgi, Phys. Rev. Lett. 63, 1917 (1989); Nucl. Phys. B 331, 311 (1990).

[16] M. Harada and K. Yamawaki, Phys. Lett. B 297, 151 (1992).

[17] M. Tanabashi, Phys. Lett. B 316, 534 (1993).

[18] M. Harada and K. Yamawaki, Phys. Rev. D 64, 014023 (2001).

[19] M. Bando, T. Kugo and K. Yamawaki, Nucl. Phys. B 259, 493 (1985); M. Bando, T. Fujiwara and K. Yamawaki, Prog. Theor. Phys. 79, 1140 (1988); N. Kaiser and U. G. Meissner, Nucl. Phys. A 519, 671 (1990). 